\title{
Tracking F-region plasma depletion bands using GPS-TEC, incoherent scatter radar, and all-sky imaging at Arecibo
}

\author{
Ilgin Seker ${ }^{1}$, Dorey J. Livneh ${ }^{1}$, Jonathan J. Makela ${ }^{2}$, and John D. Mathews ${ }^{1}$ \\ ${ }^{1}$ Communications \& Space Sciences Laboratory (CSSL), Penn State University, PA, USA \\ ${ }^{2}$ Department of Electrical and Computer Engineering, University of Illinois at Urbana-Champaign, IL, USA
}

(Received January 28, 2007; Revised December 24, 2007; Accepted December 25, 2007; Online published July 4, 2008)

\begin{abstract}
F-region plasma dynamics has been one of the main foci of the ionospheric research community for decades. The mid-latitude F-region has been considered to be relatively calm; however recent observations using highly sensitive CCD imaging systems and Global Positioning System (GPS) receivers have revealed that mid-latitude F-region mesoscale electrodynamics are more complex and this region is more active than usually assumed. Here we report combined incoherent scatter radar (ISR), imager, and GPS observations of F-region MediumScale Traveling Ionospheric Disturbance (MSTID) structures over the Arecibo Observatory in Puerto Rico. In particular, the plasma structures seen in the narrow-beam ISR cannot be understood fully without the all-sky images, which provide the context for the radar results-specifically, the spatial and temporal properties of the mesoscale structure. The GPS-derived total electron content (TEC) data provide additional information on the intensity of the MSTIDs. Here we present analysis of two specific plasma depletion events, which we prefer to call "MSTID bands". Important results on the 3D geometry of these structures were found using a newly developed observation technique. For the first time, it is shown that the southern part of MSTID bands reaches higher altitudes than the northern part (vertically tilted by $12^{\circ}$ towards magnetic south). These results give a much broader perspective on nighttime, mid-latitude F-region structure and point to new ways of interpreting these structures and how they appear in ISR results.
\end{abstract}

Key words: F-region, plasma depletion bands, GPS-TEC, ISR, all-sky imaging, MSTID.

\section{Introduction}

The Penn-State All-Sky Imager (PSASI) installed at Arecibo Observatory (AO: $18.3^{\circ} \mathrm{N}, 66.75^{\circ} \mathrm{W}$, altitude $350 \mathrm{~m}, L=1.43$ at $300 \mathrm{~km}$, dip angle $46^{\circ}$, geomagnetic coordinates $31.5^{\circ} \mathrm{N}, 8^{\circ} \mathrm{E}$ ) is a high-resolution optical all-sky imager which has been operating since April 2003. Figure 1 shows the geomagnetic field line geometry associated with the site and the coverage of the imager (limited to $150^{\circ}$ field of view) assuming an emission height of $300 \mathrm{~km}$. Data is collected at night during moon-down conditions. The imager is configured to primarily monitor mesoscale F-region processes using the $180^{\circ}$ field-of-view (FOV) primary lens and 557.7-nm, 630.0-nm, and 777.4-nm narrowband filters. The all-sky imager data for more than three years is available online (allsky.ee.psu.edu) and is being used to study various ionospheric phenomena. More details on PSASI and all-sky imaging in general are available in Seker et al. (2007).

Plasma depletions have long been observed by all-sky imagers, especially at low latitudes using the 557.7-nm, 630.0-nm, and 777.4-nm airglow emissions. A detailed review of low-latitude imaging of ionospheric irregularities is given by Makela (2006). Although low-latitude depletions have been observed using imaging techniques for over

Copyright (c) The Society of Geomagnetism and Earth, Planetary and Space Sciences (SGEPSS); The Seismological Society of Japan; The Volcanological Society of Japan; The Geodetic Society of Japan; The Japanese Society for Planetary Sciences; TERRAPUB. two decades, it is only in the last decade that the imaging technique has been applied to mid-latitudes. Recent imaging studies have revealed that the mid-latitude F-region is also active with plasma depletion bands (or so called slabs, MSTIDs), equatorial plumes, the midnight temperature maximum, spread-F, etc. (Mendillo et al., 1997; Garcia et al., 2000; Kelley et al., 2000b, 2002, 2004; Shiokawa et al., 2003a, b; Otsuka et al., 2003; Seker et al., 2007). The use of imager and radar together has been the subject of several recent papers: Pi et al. (2000) (imager, ISR, FPI), Kelley et al. (2000a) (imager, ISR), Kelley et al. (2002) (imager, ISR, GPS), Otsuka et al. (2003) (imager, MU radar, FPI). However these papers have typically been limited to comparing the radar "image" and the all-sky image by looking at them simultaneously, rather than using the two jointly to infer additional information about the structures being studied.

As will be shown in the next section, we have employed a technique which includes tracking the Arecibo radar beam in the all-sky images and displaying the radar beam on the image at the appropriate time automatically by means of a computer code that "fuses" the results from the two instruments. This technique is most useful if the radar beam is scanning in azimuth and thus can provide some spatial information to compare with the images. When GPS-TEC data is available, we include these data in the analysis. First, in Section 2.1 we describe a strong MSTID event for which radar data with fixed vertical beam was available. In Sec- 
(a)

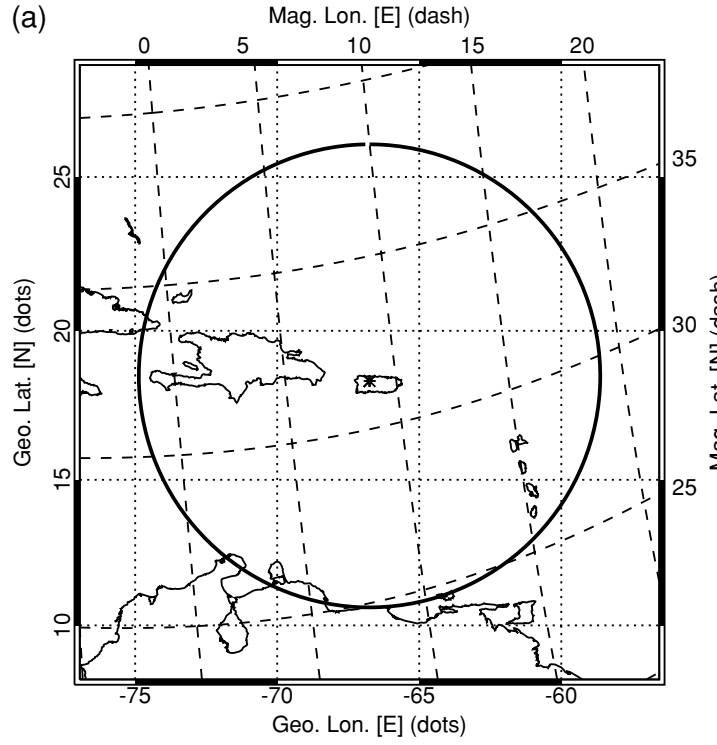

(b)

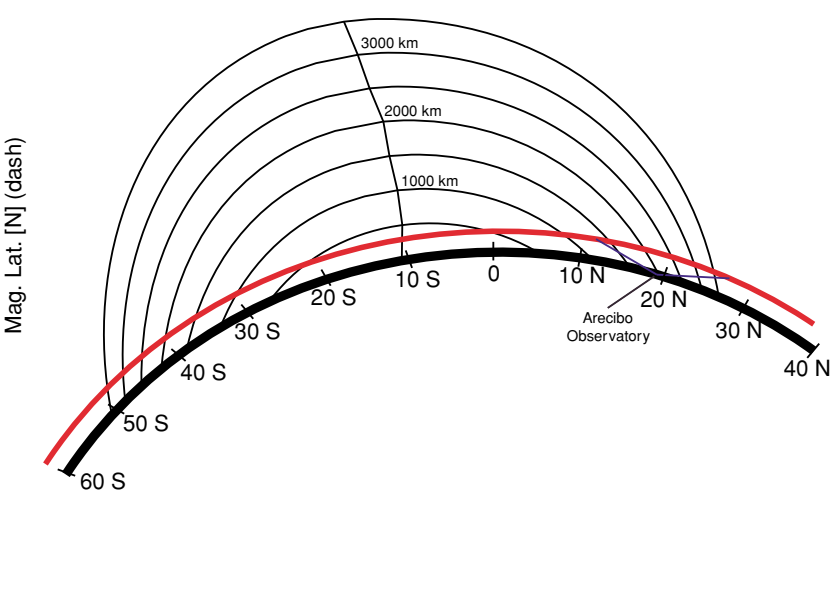

Fig. 1. (a) Geographic coordinate (latitude vs. longitude) map centered on AO showing coverage of the imager with a field of view of $150^{\circ}$ assuming an emission height of $300 \mathrm{~km}$. (b) Geomagnetic field line distribution in the magnetic meridional plane of Arecibo (latitude vs. altitude) mapping the $630 \mathrm{~nm}$ airglow depletions at $300 \mathrm{~km}$ seen in the imager to an apex height of 1500-3500 km. Altitude Adjusted Corrected Geomagnetic Coordinates (AACGM) are used.

tion 2.2 we present a weaker event for which radar data was available in the azimuth-scanning mode. In Section 3 we compare the results from the ISR and imager by the aforementioned technique and provide further insight by making GPS-TEC versus imager comparisons. We then discuss the benefits of using ISR, GPS-TEC, and all-sky imager data in collaboration instead of using them separately. We conclude in Section 4 by emphasizing particular results of importance highlighted by this study.

\section{Results}

\subsection{Fixed beam (22-23 March 2004)}

Figure 2 shows a typical ISR power profile mode result from the Arecibo Observatory (AO) operating in a fixedzenith observing mode on 22-23 March 2004, a geomagnetically quiet night (see Fig. 4). Figure 2(a) shows a "typical" midnight collapse from 2300-2800 LT (Nelson and Cogger, 1971; Herrero and Meriwether, 1980; Colerico et al., 1996; Mendillo et al., 1997). The rectangle highlights the vertical features occurring during the collapse, and is expanded in Fig. 2(b). The dashed lines at $250 \mathrm{~km}$ (the assumed height of the 630.0-nm emission) in Fig. 2(b) denote the times at which 60-second integration time images were obtained with PSASI. Figure 3 shows the all-sky images at $630.0 \mathrm{~nm}$ from PSASI, corresponding to the numbered features in Fig. 2(b). The individual images are flat-fielded and mapped to geographical coordinates (the grid size is $200 \mathrm{~km} \times 200 \mathrm{~km}$ yielding an image of about $1200 \mathrm{~km}$ on a side). The frames reveal distinct MSTID bands aligned from the northwest to southeast. As the radar beam on this night was fixed at zenith, the ISR results correspond to a point roughly at the center of these images. The parameters calculated from the unwarped all-sky images are given in Table 1. The nighttime F-region electrodynamics and thus the MSTIDs seen in the all-sky images are presumed to be described by the so called Perkins Instability
Table 1. The horizontal parameters (calculated from the allsky images) for the event of 22-23 March 2004.

\begin{tabular}{ll}
\hline Tilt angle & $20^{\circ}-40^{\circ}$ westward from north \\
Depletion width & $50-250 \mathrm{~km}$ \\
Wavelength & $100-200 \mathrm{~km}$ \\
Horizontal speed & $100-200 \mathrm{~km} / \mathrm{h}$ \\
Period & $\sim 1$ hour \\
Intensity variation & $\sim 5-15 \%$ \\
\hline
\end{tabular}

equations (nighttime F-region appropriate electron/ion momentum and continuity equations along with divergence of current density equals zero) (Perkins, 1973; Garcia et al., 2000; Kelley and Makela, 2001; Zhou et al., 2006; Zhou and Mathews, 2006). Numerical solutions of the Perkins equations for simulation of nighttime F-region electrodynamic processes indicate that the depletion/enhanced structures (observed in the all-sky camera) develop northwestto-southeast aligned "wave fronts" that propagate to the southwest in the northern hemisphere. These simulations are valid when the F-region is electrodynamically decoupled from the E-region and the conjugate ionosphere (Zhou et al., 2005, 2006; Zhou and Mathews, 2006). In practice, the numerical simulations are most valid when geomagnetic activity is low and large-scale E-fields are small. However we note that observations have shown strong E/F-region electrodynamic coupling during low $\mathrm{Kp}$, intense spread-F events (Mathews et al., 2001; Swartz et al., 2002; Kelley et al., 2003a).

An extensive examination of the $\sim 35$ hours of observations on 22-23 March 2004 using a high-pass filter (nonringing) shows the presence of $\sim 1$ hour period "waves" that are apparent from the $\mathrm{E}$ region through the top of the Fregion and persist through the day (Livneh et al., 2007). The dark bands seen in the all-sky images presented in Fig. 2(a) and 3 appear to be part of the continuum of these $\sim 1 \mathrm{hr}$ 


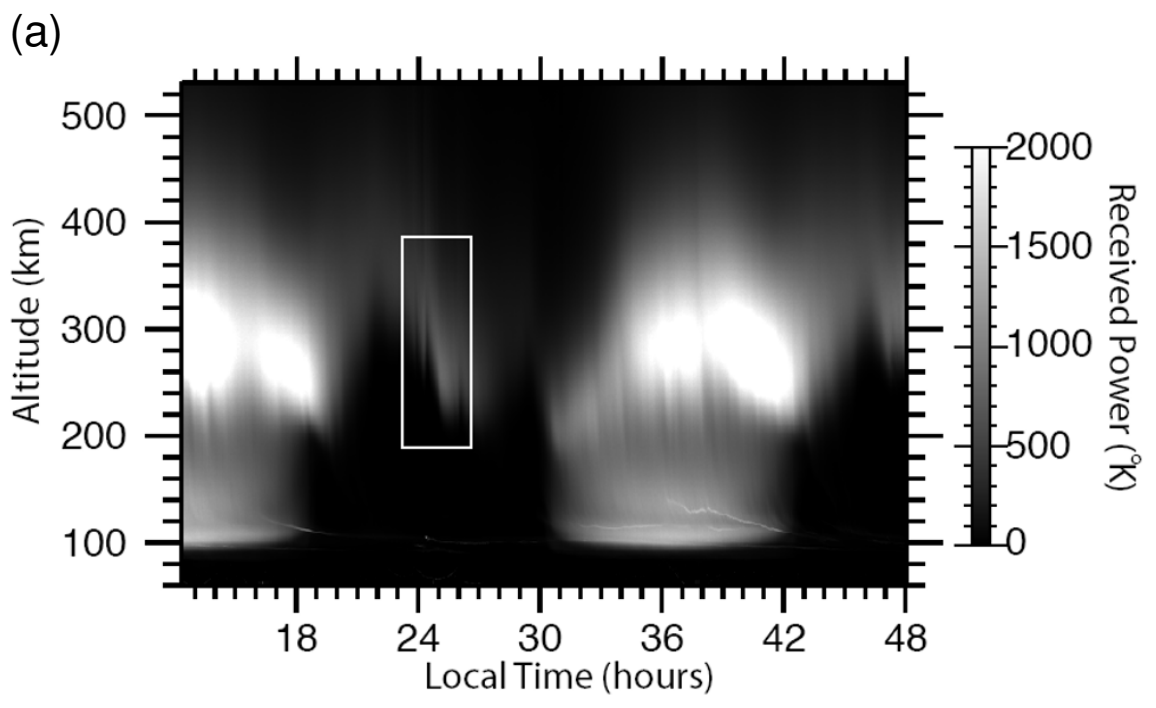

(b)

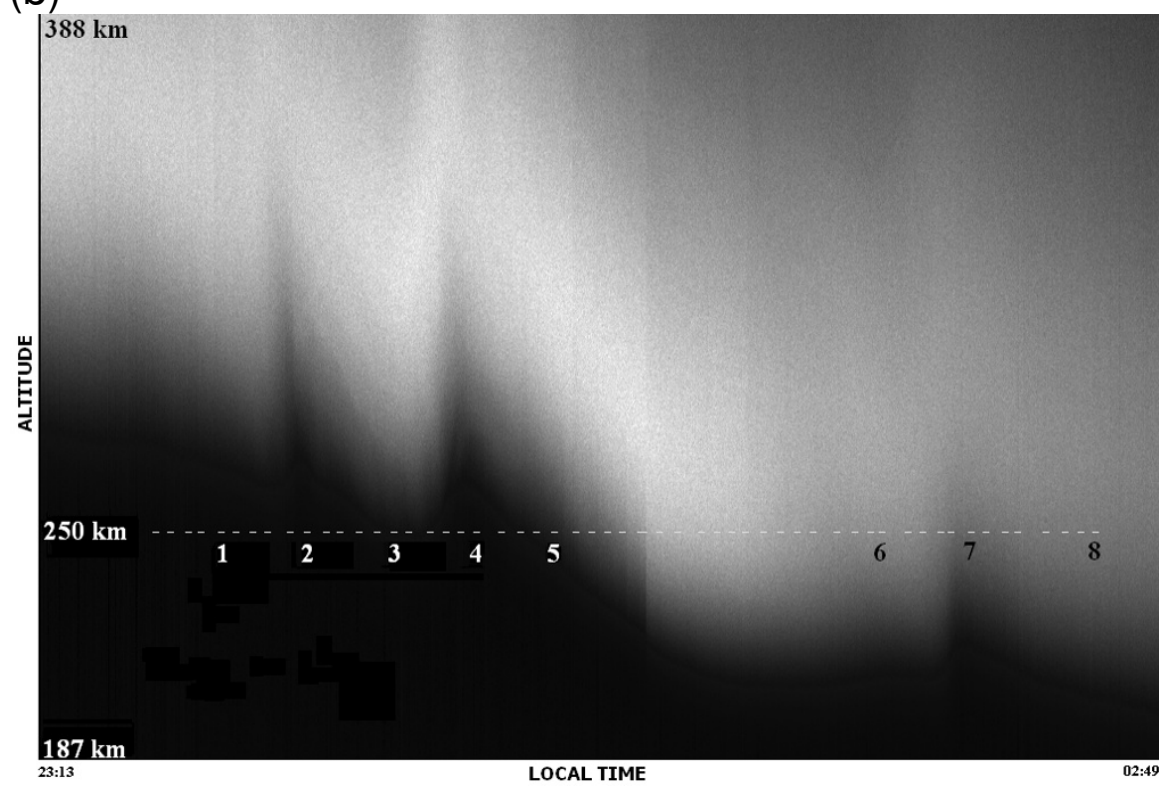

Fig. 2. AO observing program T1892 providing vertical ISR signal temperature (Mathews, 1984, 1986) results from 22-23 March 2004. (a) shows a typical midnight collapse from 23-28 hours. (b) is the enlarged version of the rectangular region. The numbers correspond to the allsky images in Fig. 3.

period waves as explained in Livneh et al. (2007). The properties and possible origins of these waves are extensively discussed in Livneh $e t$ al. We note that Fig. 2 of Vadas (2007) gives Acoustic-Gravity Wave (AGW) dissipation heights below $200 \mathrm{~km}$ for cold thermosphere (solar minimum) conditions whereas we observe these waves at $\sim 250 \mathrm{~km}$ in the camera results and to altitudes in excess of $700 \mathrm{~km}$ in the ISR results. This leads us to note that the collisional coupling between the neutral atmosphere and the Fregion plasma weakens rapidly with increasing altitude and that, as pointed out above, the electrodynamics of waves in the nighttime F-region must obey the electrodynamic equations given by Perkins (1973) and elucidated in Zhou et al. $(2005,2006)$ and Zhou and Mathews (2006). In particular, to the extent to which the F-region is decoupled from the Eregion, the propagation of wavelike features in the F-region is enhanced to the southwest (and northeast) while damped to the northwest/southeast thus providing a filtering mecha- nism as well as a possible amplification (growth/instability) mechanism (e.g. Taylor et al., 1998) that certainly influences what we observe with both the ISR and the all-sky camera systems. Further, we note that the F-region plasma is incompressible for motions parallel to the geomagnetic field. Thus, as the neutral atmosphere AGW forcing of the plasma decouples at the base of the F-region due to decreasing collision frequency (and also due to altitude progressive AGW losses), the field-aligned plasma motions at the decoupling altitude are imposed on the entire plasma along that flux tube.

Comparison of Fig. 2(b) and Fig. 3 reveals that there is a good agreement between the ISR and all-sky results which complement each other by providing vertical and horizontal information respectively, revealing the midnight temperature maximum driven midnight collapse to have the airglow depletion wave-structure embedded in it. For example, images 3, 4, and 5 track the Fig. 2(b) radar results almost ex- 


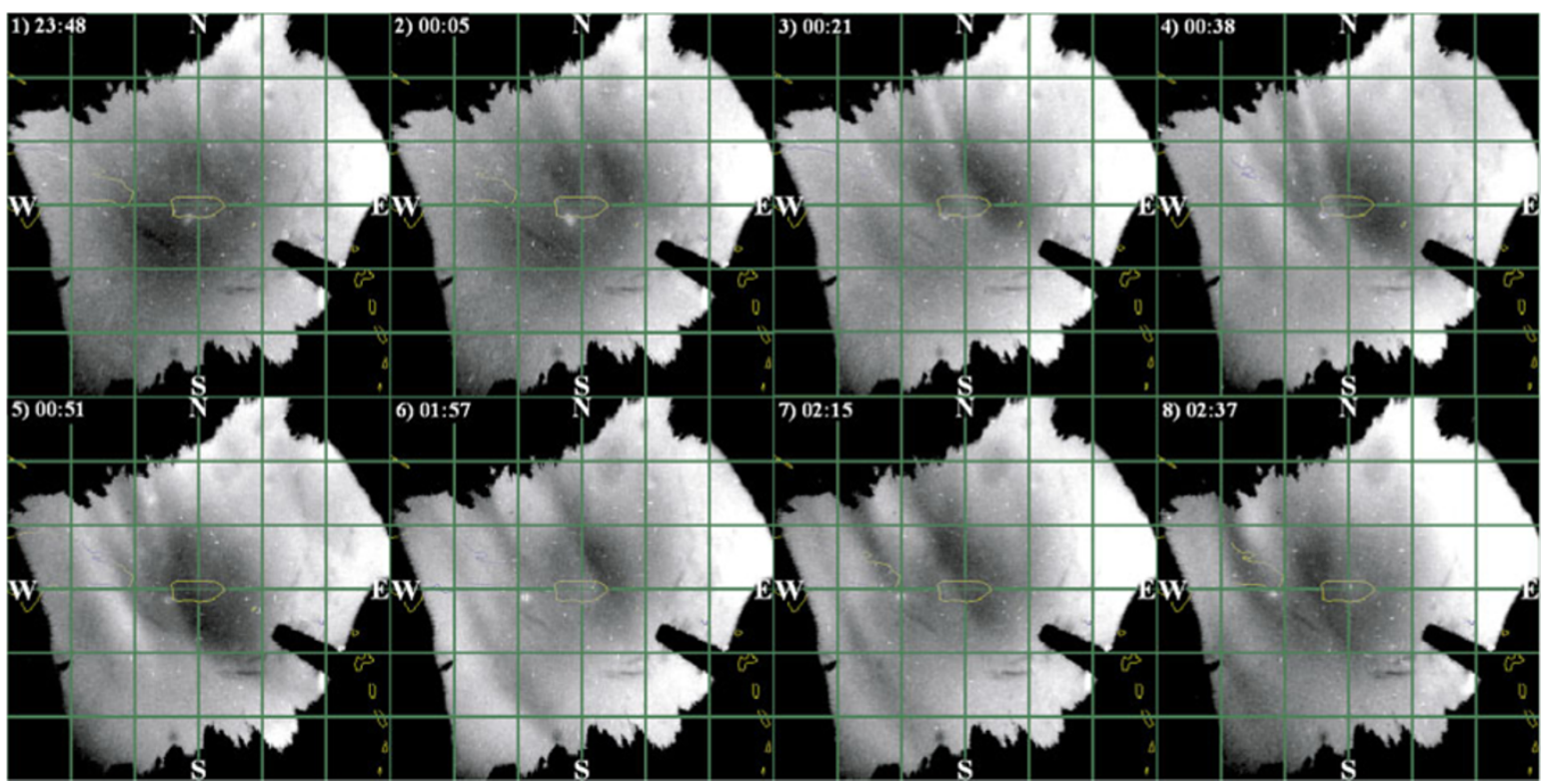

Fig. 3. Allsky imager $630 \mathrm{~nm}$ frames from PSASI corresponding to the 1-8 numbered features in Fig. 2(b) and showing depletion bands moving to the south-west. The streaks at the south-east part of the images are due to the dirt on the telecentric lens, the bright region at north-east of each image is due to the city lights of San Juan, and the black rectangular area is due to a shield blocking the lights on one of the AO towers.

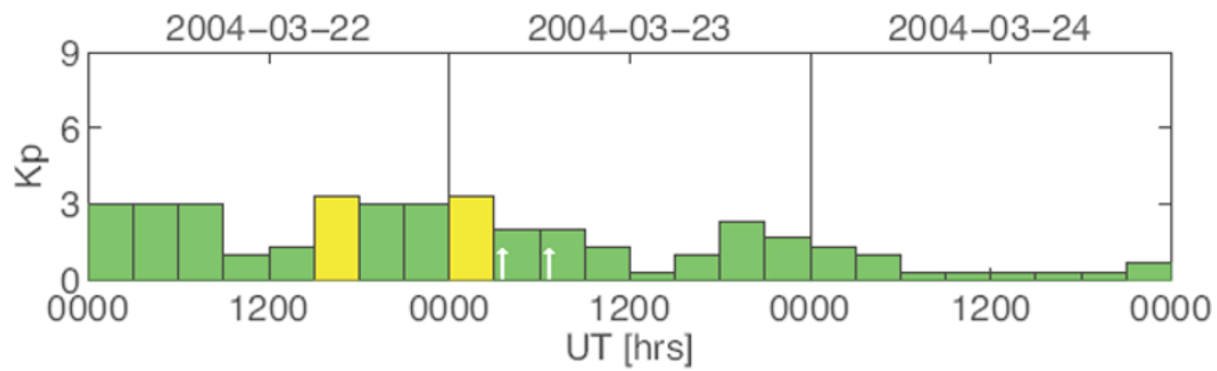

Fig. 4. Kp index for 22-24 March 2004 (UTC). The duration of the event is indicated by two arrows.
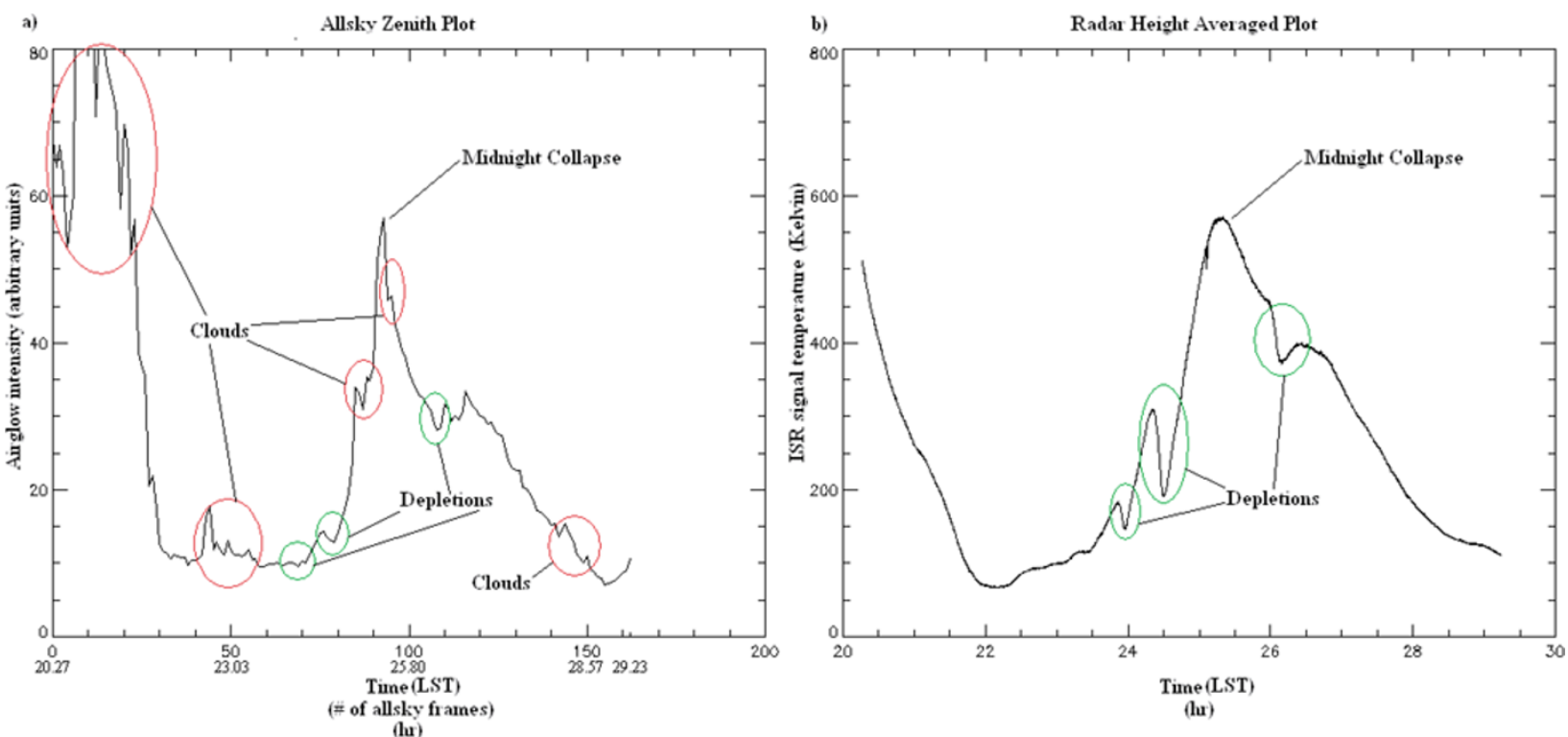

Fig. 5. 22-23 March 2004 from 2016 to 0514 LST. (a) Allsky Zenith Plot (averaging intensity over $3 \times 3$ pixels). Depletion bands and clouds are shown. The intense area centered at frame 95 (0130 LST) is the well-known "Midnight Collapse". (b) Corresponding ISR signal power plot averaged through 210 to $330 \mathrm{~km}$. Depletion bands and midnight collapse are shown. 
actly with the progression from F-region low (bright band overhead), high (dark band overhead), and lowering (dark band passing). Another straightforward way to see the relationship between the airglow depletions and ISR data is to plot the zenith data of the all-sky images and compare them with the airglow emission height-range-averaged ISR data for the corresponding time interval. We show the results of doing this in Fig. 5, which reveals a strikingly similarity between the two datasets. Specifically the three depletion bands (corresponding to the frames 2, 4 and 7 in Fig. 3) are apparent in both plots.

\subsection{Swinging beam (15-17 June 2004)}

Although data from the radar with beam swinging (azimuth scanning) provides more information such as electric fields, without the imager data providing the horizontal structure, the medium-scale features are blurred. Thus, the imager and radar data should be used together to analyze the plasma features when the radar beam is swinging. Further, with the addition of GPS-TEC data, additional insight into the properties of the F-region structure can be gained. Figure 6 shows the AO world day ISR data from 14-18 June 2004. In contrast to the March 2004 case studied above, the radar beam is at a $15^{\circ}$-zenith angle and is swept $0^{\circ}-360^{\circ}-$ $0^{\circ}$ cyclically with an approximate period of 16 minutes per half-cycle. As the beam sweeps through different horizontal regions of the ionosphere in this way, the resultant ISR data shows periodic vertical features (Aponte et al., 2000; Makela et al., 2001b; Kelley et al., 2003b). Figure 6(a) and $6(\mathrm{~b})$ show the profiles covering approximately two days each, all of which were geomagnetically quiet $(\mathrm{Kp} \sim 2)$ as shown in Fig. 9. The "midnight collapse" feature (approximate duration is indicated by the bar at the bottom of each figure) can be seen during each of the four nights, especially on 14-15 June. Even though the azimuth scanning may blur the details, spike-like depleted plasma features can still be observed clearly during the nights of June 15-16 and June 16-17. Figure 7 shows the enlarged version of these structures on 16-17 June in the rectangular box highlighted in Fig. 6(b). The two long lines at the top show the time intervals of the two separate MSTID bands passing over the radar. Again, the dots and the letters in Fig. 7(a) correspond to the available all-sky images from the night and to the images shown in Fig. 8, respectively. The squares/circles, the letters A and B, and the short lines at high altitudes are placed to show the effects of 3D geometry on the azimuthscanning ISR data and their meaning will be explained in the discussion section. In addition, an azimuth angle vs. time plot is inserted above Fig. 7(a) and 7(b) to emphasize the effect of beam swinging on the ISR data.

The peak electron concentrations calculated from the ISR and especially the Ramey ionosonde seem to be affected by these MSTIDs embedded in the midnight collapse structure as Fig. 6(c) clearly shows small oscillations (with $\sim 1 \mathrm{hr}$ period) between 2500-3000 LT. Since the ISR beam is swinging, the mesoscale slower variations are modulated by the rapid oscillations (with 16 min. period) that are due to the beam azimuth-scanning across large-scale gradients in the ionosphere, and thus it is harder to see the waves with $\sim 1 \mathrm{hr}$ period in the ISR peak electron concentrations (blue line) compared to the ionosonde results (red line). From this result, it can be concluded that these MSTIDs indeed cover a wide range of altitudes. Since the smaller scale structures inside the depletions/enhancements are thought to be Bfield aligned, they are most likely B-field aligned "slabs" of the ionosphere that are raised (airglow depletion) or lowered (airglow enhancement). We demonstrate this in the next section. Figure 8 shows the unwarped all-sky images from PSASI corresponding to the times labeled with lower case letters in Fig. 7(a). The white square at the center shows the zenith of the imager and radar. The other square shows the north direction at a zenith angle of $15^{\circ}$, which corresponds to the radar beam location with $0^{\circ}$ azimuth angle. The arc shows the motion of the beam during the 60-second exposure time of the images, and the arrowhead indicates which way the radar beam is rotating. The yellow contour shows the island of Puerto Rico $(\sim 150 \mathrm{~km} \times 50 \mathrm{~km})$.

In Fig. 10 we present total electron content (TEC) data obtained along the ray-path from the GPS receiver at Arecibo Observatory to various GPS satellites during the night. The positions of the satellite-to-receiver ray path at $350 \mathrm{~km}$ is shown in these allsky images. This technique has been used extensively in studying the effects of ionospheric depletions (e.g., Makela et al., 2000, 2001a). Doing so again demonstrates the fact that the dark bands seen in the $630.0-\mathrm{nm}$ emission are caused by the lack of electron concentration in the structure, rather than a localized uplift in the ionosphere. This is an important distinction, as the intensity of the 630.0-nm emission is dependent both on the concentration of the ionosphere as well as its height (Link and Cogger, 1988). Thus, without the additional information provided by the ISR and GPS, there is an ambiguity in interpreting the $630.0-\mathrm{nm}$ emission.

\section{Discussion}

Recent studies have shown that the short-duration features regularly seen in ISR results as shown in Fig. 2(b), are not a direct result of the midnight collapse (MTM) event, but rather a manifestation of the $\sim 1 \mathrm{hr}$ periodic wave process that extends through the whole ionosphere (Livneh et al., 2007). However, these features appear to be more intense during the midnight collapse period especially when the geomagnetic activity is low. An examination of Kp values in Figs. 4 and 9 in comparison with the strength of plasma MSTID activity in Figs. 2(a), 6(a) and 6(b) supports the idea that these depletion bands tend to appear more often when the $\mathrm{Kp}$ is lower (as in $2^{\text {nd }}$ and $3^{\text {rd }}$ nights of Fig. 6(a) and $6(b)$ ). Although, there has been no previous in-depth study on the correlation between geomagnetic activity and mid-latitude plasma depletion bands, one study showed that when the solar activity is high, the equatorial plumes are observed more often and more toward mid-latitudes (Sahai et $a l ., 2000)$. From that and the correlation between Kp and plumes/depletion bands observed by PSASI (e.g. in Seker et al., 2007), it could be inferred that as the geomagnetic activity becomes more disturbed, it becomes more likely to observe equatorial plumes reaching AO latitudes instead of these MSTID bands. More studies on this need to be done in order to understand the dynamics of these phenomena.

From ISR data alone, one can infer some properties of these depleted regions such as vertical extent and plasma- 

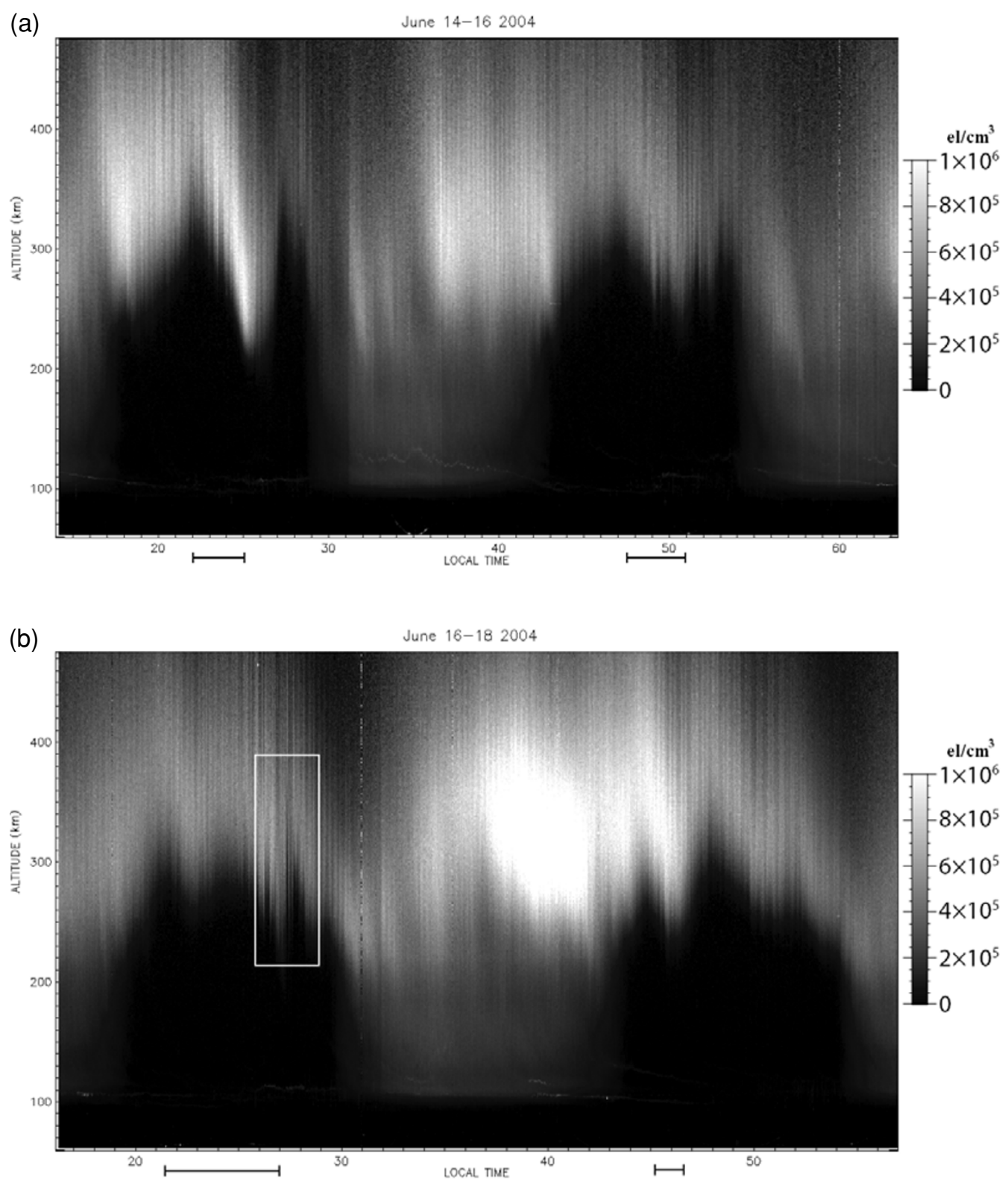

(c)

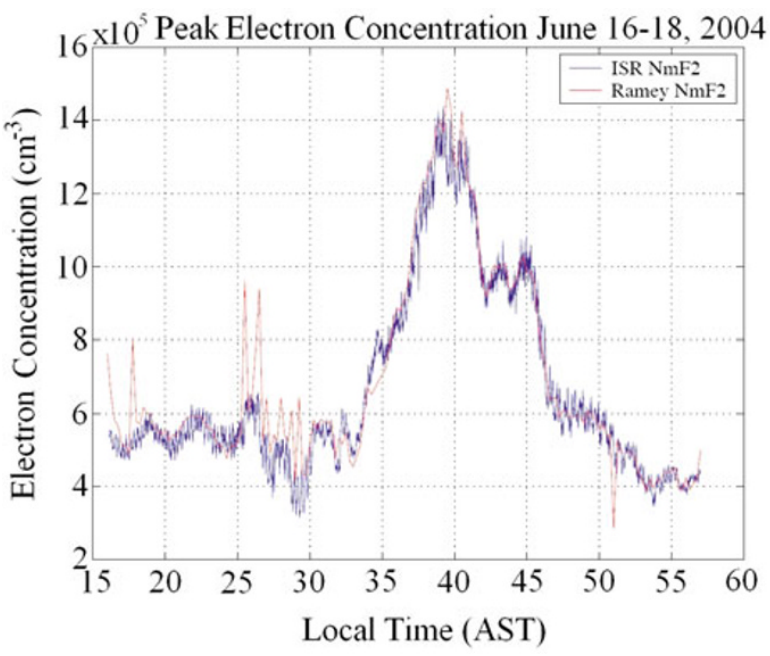

Fig. 6. AO world day ( $15^{\circ} \mathrm{ZA}$ in continuous azimuth scans) ISR data from (a) 14-16 June 2004, (b) 16-18 June 2004. The time of midnight collapses are indicated with lines at the bottom of the figures. (c) Peak electron concentration from AO ISR and the Ramey ionosonde. The ionosonde result outliers are due to scaling errors as a result of weak signal returns. (source: N. Aponte) 
(a)

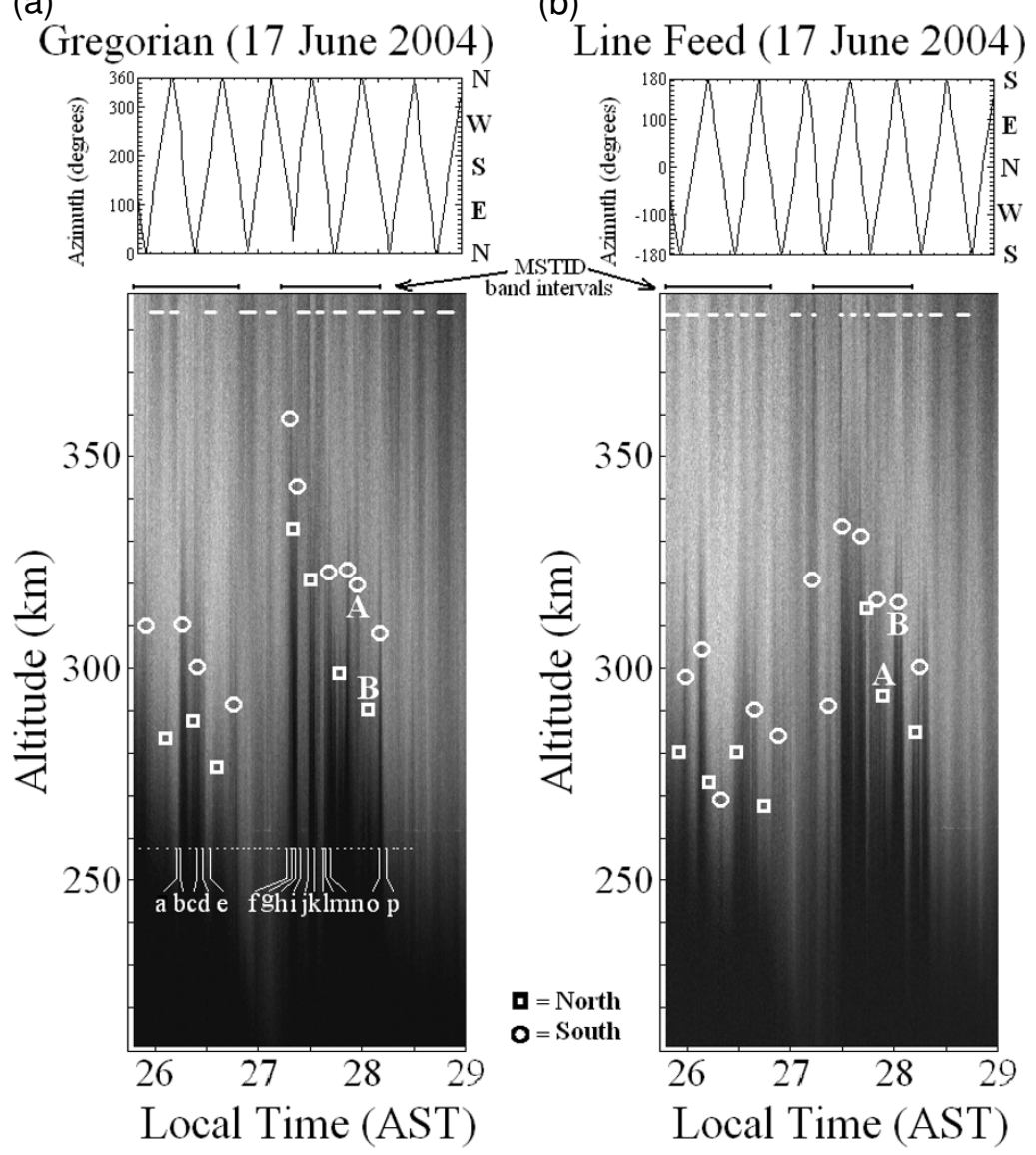

Fig. 7. (a) Gregorian Data in the rectangle of Fig. 6(b) in more detail. The dashed lines around 260 km correspond to the available allsky images from that night and the letters correspond to the frames shown in Fig. 8. The allsky frames corresponding to letters A and B are shown in Fig. 11. The long black lines at the top represent the two MSTID bands passing over. The white lines at high altitudes show the neighbor bands, and the squares (circles) correspond to the height of the spikes when the beam is positioned at northern (southern) parts of a depletion band. Azimuth angle variation is also shown. The very distinct "edges", for example at $27.5 \mathrm{hrs}$, reveal very sharp edges in the F-region plasma distribution. (b) Corresponding data from the linefeed that is pointed $180^{\circ}$ from the Gregorian feed.

drift velocity (if dual-beam azimuth scanning is employed). However the corresponding all-sky images clearly reveal that the ISR results represent a single-point altitude/time sequence of a rather complex mesoscale structure that is, in the cases presented here, wavelike in appearance. That is, the ISR results do not fully characterize the horizontal extent of these structures. Notice how well the individual images in Fig. 3 agree with the numbered profiles in Fig. 2(b). In the ISR data corresponding to Frames 3 and 6 in the imager data, we see that the ionosphere base is lower resulting in higher recombination. This leads to a brighter emission, as seen in the allsky imaging data. In Frames 2, 4 and 7 dark structures are seen to be overhead of the Arecibo Observatory and a corresponding depletion (mostly due to uplift) is seen in the ISR data. It is the fusion of the two data sets that begin to reveal the full extent of what is happening. These depletion regions appear to obey the well-known linear Perkins state equations of the ionosphere, since the model and the observation agree with each other well in terms of orientation and propagation direction (Perkins, 1973; Zhou et al., 2006).

The June 2004 event is similar to, but weaker than, the March 2004 event and similar conclusions can be drawn. However, the ISR beam is in azimuth-scanning mode, and although the scanning provides E-field and drift information, it distorts (aliases) the plasma depletion bands seen in the radar image and makes it more difficult to compare the imager and radar results. In order to show the directionality of ISR data, it is possible to create the azimuthal maps as used in Aponte et al. (2000). An azimuthal map is a polar plot displaying each column of the ISR data with respect to angle; so each azimuthal map shows $16 \mathrm{~min}$. of data (a full circle). Here, we employ a different technique to visualize the directionality of ISR data which also allows us to compare the imager and ISR results. As shown in Fig. 7(a) and Fig. 8, by identifying the beam location precisely in the images, one can track the MSTID bands very well even when the beam is scanned. For example, from the allsky images in Frames a, e and $\mathrm{f}$ of Fig. 8, it is seen that the ISR beam is pointing at the MSTID band while the ISR data shows uplifted/depleted regions. Similarly, in frames c, h, k and $\mathrm{n}$, the ISR beam is pointing at an enhanced region while the ISR data shows enhanced electron concentration. Most importantly, there are the transition regions (edges) which prove how well the two data sets agree with each other. For example, frames $\mathrm{d}$, i and $\mathrm{k}$ in both Figs. 7(a) and 8 correspond to the regions where the beam is about to transit from depletion to enhancement; and the frames $b, j$ and $m$ cor- 


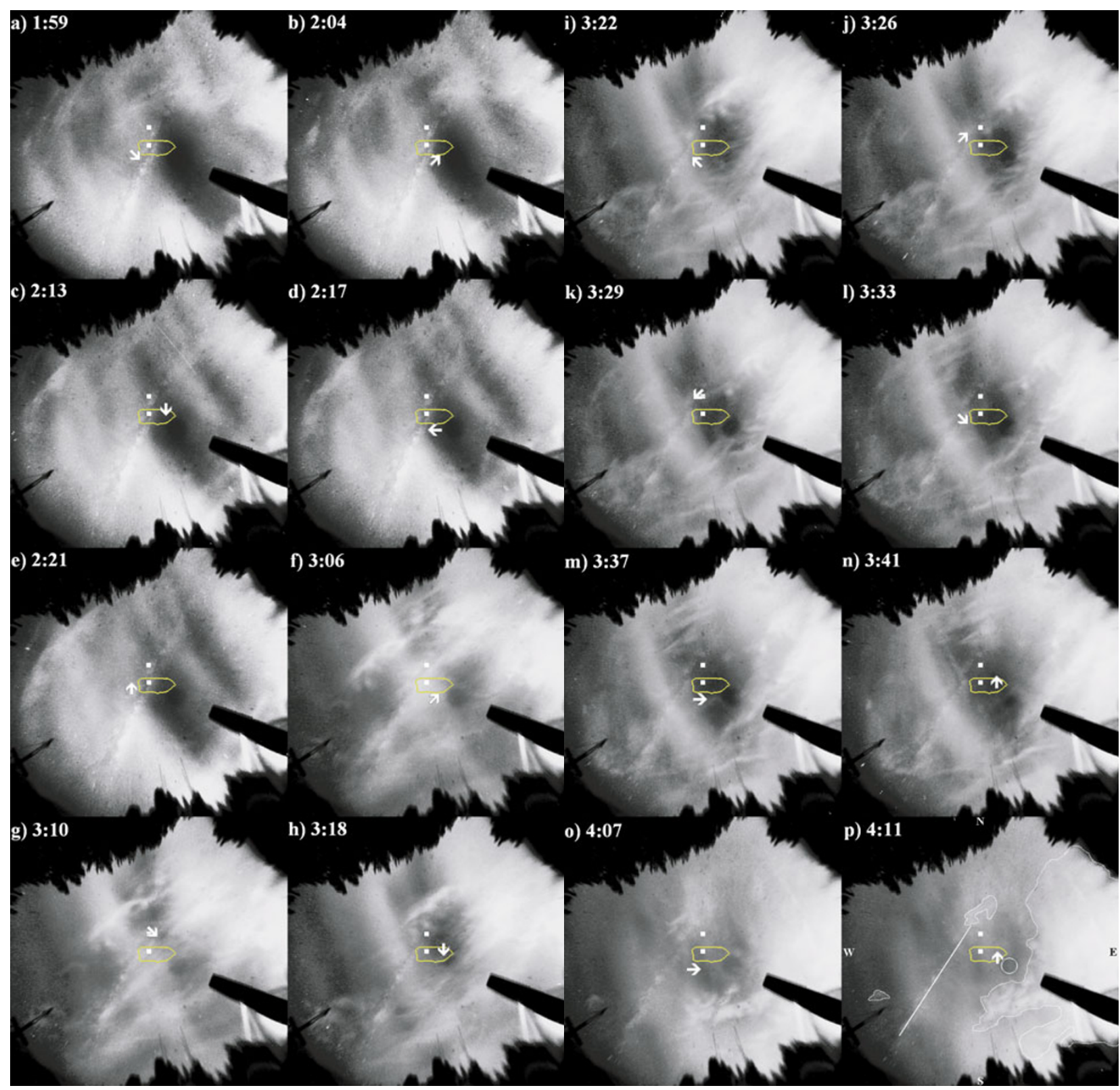

Fig. 8. The unwarped $60 \mathrm{sec}$ time-averaged allsky images from PSASI corresponding to the times labeled with letters in Fig. 7(a). The arrow shows the ISR beam location and direction, and the two dots correspond to the north and zenith directions. The yellow contour shows the island of Puerto Rico $(\sim 150 \mathrm{~km} \times 50 \mathrm{~km})$. The images are enhanced by removing the stars using a median filter and performing histogram equalization in order to show the weak plasma depletion event with a better contrast. The night is somewhat cloudy, especially around $3 \mathrm{am}$. Features that can be seen in the images along with the geographical directions are indicated in the last frame p. Clouds are contoured and the Milky Way disk is shown with a line. The constant relatively darker disc region (encircled) is the dirt on the lens.

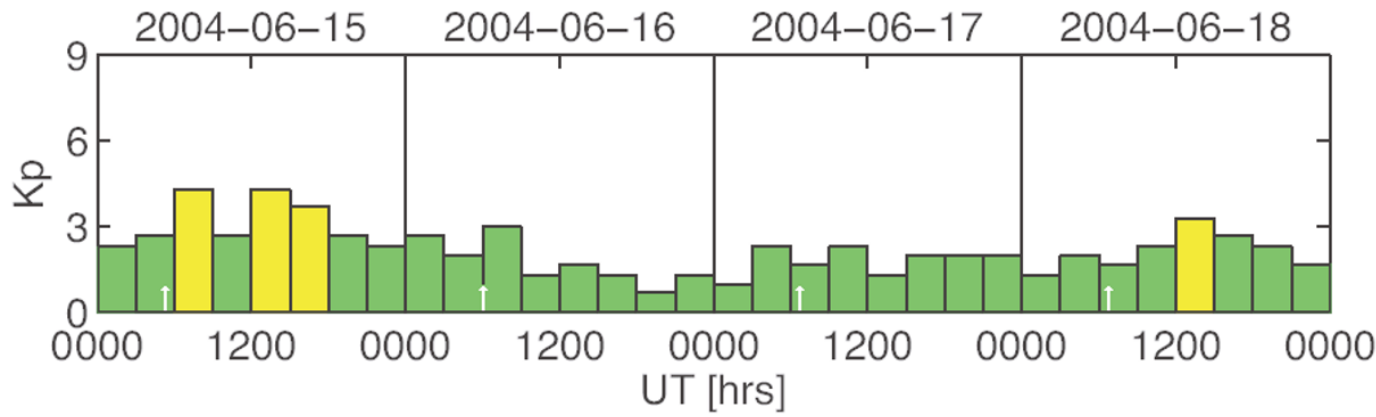

Fig. 9. Kp index for June 15-19 2004 (UTC). The midnight collapse times for each night is indicated with an arrow. 
(a) TEC from GPS Satellite 1
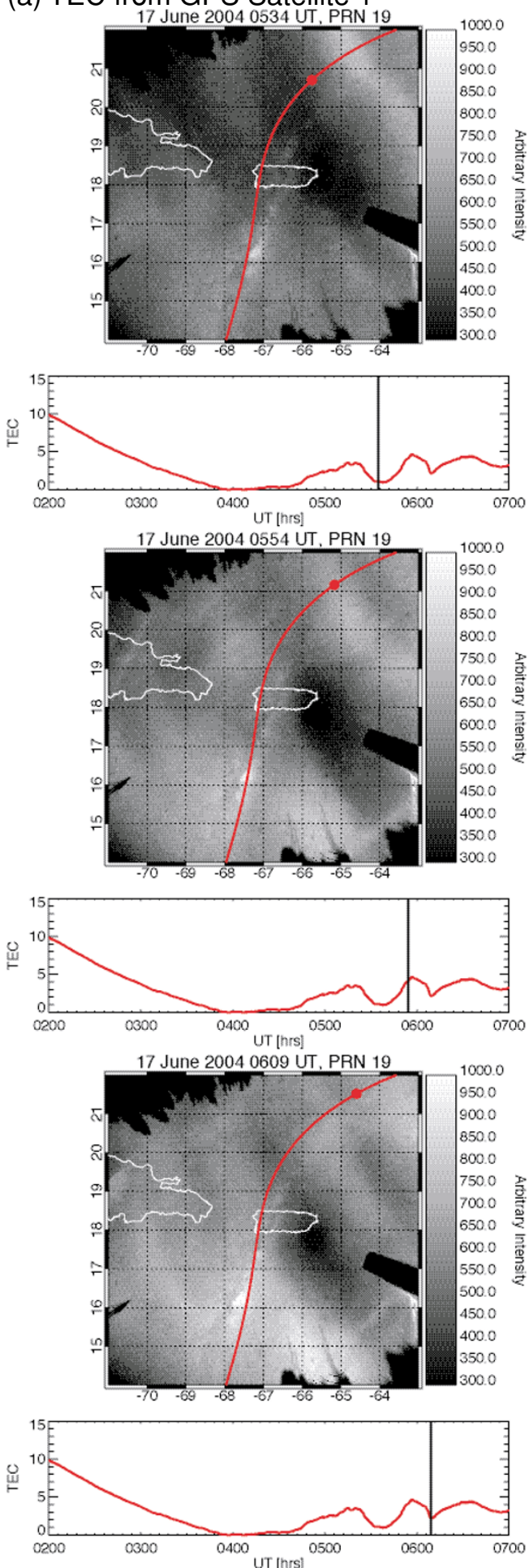

(b) TEC from GPS Satellite 2
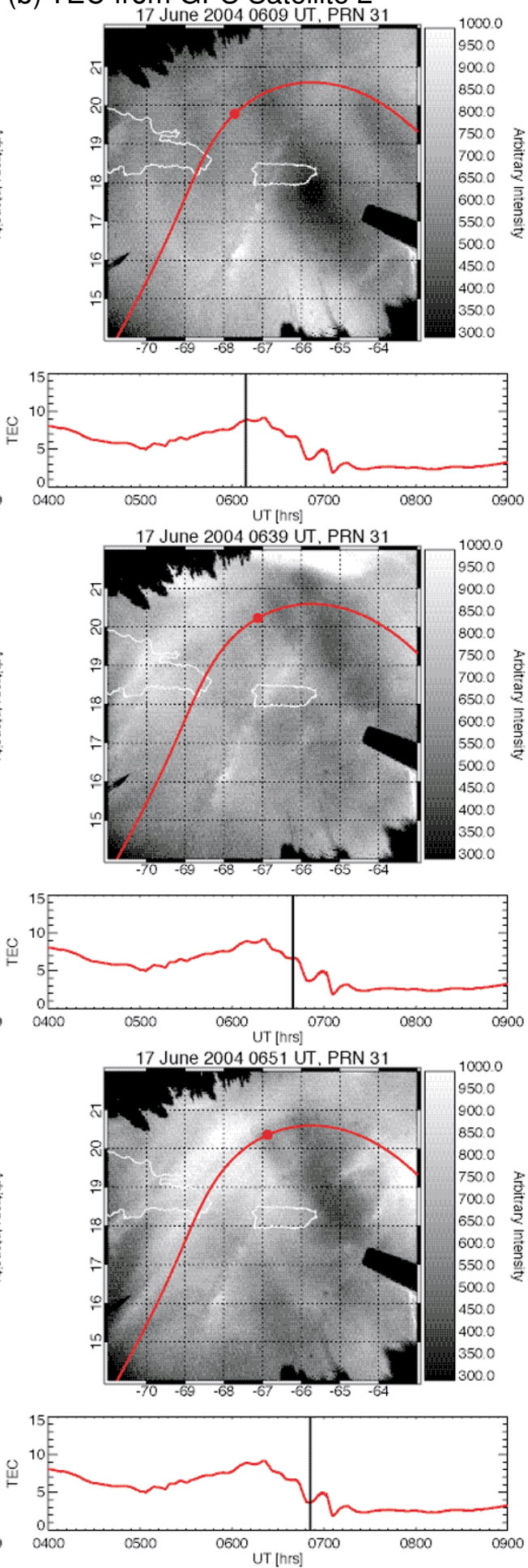

Fig. 10. 16-17 June 2004 TEC data from two different GPS satellites overlapped with the allsky images. The vertical lines on the TEC plot for each frame denotes the time at which the allsky image data was collected.

respond to the regions where the beam is about to transit from enhancement to depletion. It is important to note how critical the beam position relative to the position of MSTID bands is for the ISR results. Notice that even if the beam is slightly off the depleted region (as in Fig. 8, frame $\mathrm{j}$ ), the ISR shows no evidence of the features which points at how sharp the gradients at the edges of the plasma depletion bands are (Makela et al., 2001a). Clearly the very narrow radar beam ( $\sim 1-\mathrm{km}$ width at $300-\mathrm{km}$ range) gives only very local information.

Moreover, a MSTID band might show up as multiple narrow bands in the ISR image since the beam is not stationary and the banded structure is moving, as is the case here in Fig. 7(a). This method also reveals which spike-like features in the ISR results are created by the same MSTID band and which are not. For example, there are several spikes in 
the ISR data for each of the two depletion bands, shown by the long black lines at the top of the plot. That is, the thickness and spacing of the spikes vary for a single band due to the relative motion between the swinging radar beam and MSTID bands. This is demonstrated in a parallel paper (Seker and Mathews, 2008) using a 3D model that is based on the observations. Another common feature of the swinging beam data is the fork shaped spike pairs (e.g., indicated with "A" and "B" in Figs. 7(a) and 7(b)). The time between each leg of the pair varies. Comparison with the allsky images reveals that these spike pairs are the artifacts of the observation of the northern parts of a band with swinging beam. Since the beam swings around geographic north, when the depletion band is located slightly west of north, the beam passes through this MSTID band twice, moving in opposite directions, and that creates the fork with legs appearing close to each other. Similarly, when the depletion band appears further to the west (after it propagates further south-west), that would create a larger time delay between two legs. The beam usually catches the band at south-east also, but since there is no beam direction reversal south (north for line feed), this results in a single thick spike in the ISR data. This also explains the reason of seeing a double-fork and a thicker spike often in series. This double-fork feature is also demonstrated in Seker and Mathews (2008) using a 3D model.

Similar conclusions are reached with the imager-TEC comparisons. Specifically, the GPS satellites provide data along a single look angle that changes continuously as the satellite moves across the sky. That is, the line from the GPS satellite to the receiver at AO is not vertical unless the satellite is exactly over AO. Thus, considering that the plasma depletion bands also have a vertical extent, the MSTID bands affect TEC results to various degrees depending on the relative orientation of the band and the look angle (Makela et al., 2001a). For example, the TEC results are expected to drop more when the line of sight is aligned with the field line. Similarly, the TEC results also would not show any gradient when the F-layer moves only along the line of sight without changing its shape or thickness. In order to better understand the structure in the TEC data, use of all-sky images may reveal that TEC variations are due to, say, large-scale versus small-scale ionospheric structures. Close examination of Fig. 10(a) reveals that as the first set of MSTID bands pass by at around 0530 UT, the TEC drops from $\sim 4$ TEC units to $\sim 1$ TEC unit, then increases to $\sim 5$ TEC units and drops back to $\sim 3$ TEC units. From the all-sky frames it is clear that these oscillations with sharp gradients are due to local plasma depletion bands, whereas the TEC (as well as ISR) information confirms that the bands are mostly due to depletions in electron concentration along a wide range of altitudes of the whole ionosphere, rather than simply uplifting of it as was the case in Fig. 2(a). The MSTID bands embedded in the midnight collapse (which is at $\sim 0600$ UT) causing sharp TEC gradients can be observed well in Fig. 10(b). Also, although not observed in Fig. 10(b), a single MSTID band could result in multiple oscillations in the TEC plot due to the satellite's curved track moving into and out of the depleted region. This is another example of the importance of the spatial in- formation gained from the allsky images.

Another interesting point that surfaced from this study is the differences in intensity variations of the data recorded by each instrument. The ISR data varies $\sim 10-15 \%$ if averaged over whole ionospheric heights, and $\sim 15-30 \%$ if averaged only between $210 \mathrm{~km}$ and $330 \mathrm{~km}$. The allsky imager data (around $250 \mathrm{~km}$ ) varies $\sim 10-15 \%$. On the other hand, the GPS-TEC varies by a large percentage of $\sim 50-70 \%$. These differences may yet yield additional insight into the processes involved. However, it is necessary to note that each of these instruments "sees" the ionosphere very differently and so the possible significance must be regarded with caution.

One major advantage of using ISR (in scanning mode) in conjunction with the all-sky images is that one can infer information about the vertical structure of the bands at different locations within the band itself. For example the depletion at point $g$ of Fig. 7(a) extends further vertically than the depletions at points $\mathrm{k}, \mathrm{n}$ and $\mathrm{o}$. The squares (circles) in this figure represent the points in time when the ISR beam was pointing at (geomagnetically) northern (southern) parts of a band. The selection between a circle and a square is made by finding the location of the ISR beam relative to the band in the corresponding allsky image and assuming the slab remains approximately fixed between each allsky frame $(\sim 4 \mathrm{~min})$. It should be kept in mind that the azimuthal locations of the squares (circles) will only be to the NW (SE) when the structure is directly over the radar. The altitudes of the squares/circles are found by applying an intensity threshold to the radar data and they show the maximum heights of the depletions (the upper limit of the slabs). We found that a constant threshold of $10 \%$ of the average maximum electron concentration best defines the bottom-side of the F-region when there is no depletion. On the other hand, the calculated heights of the depletion peaks match the observed heights the most when the threshold is taken to be $70 \%$ of the average maximum. Since we are interested only in the depletion heights, we used a $70 \%$ threshold. It is apparent in the figure, that the circles are always higher than the nearby squares (as also demonstrated in Seker and Mathews (2008) using a 3D model), meaning that the southern part of a slab reaches higher altitudes than the northern part. This is further confirmed by comparing the Gregorian and line feed data which let us compare two different points in space simultaneously. Figure 11 shows the allsky frames corresponding to the letters A and B in Figs. 7(a) and 7(b). G and L represent the Gregorian and line feed, respectively. In Fig. 11(A), the Gregorian beam is at the southern part of the MSTID band, whereas the line feed beam is at the northern part. And as expected, the corresponding spike in Fig. 7(a) (labeled with circle A) is at a higher altitude than the corresponding spike in Fig. 7(b) (labeled with square A). The beams switch positions in Fig. 11(B). The Gregorian beam is now at the northern part of the MSTID band, whereas the line feed beam is at the southern part. So, the corresponding spike in Fig. 7(a) (labeled with square B) is at a lower altitude than the corresponding spike in Fig. 7(b) (labeled with circle B). This is an important result, since it suggests that the MSTID bands are tilted vertically with northern component of the deple- 


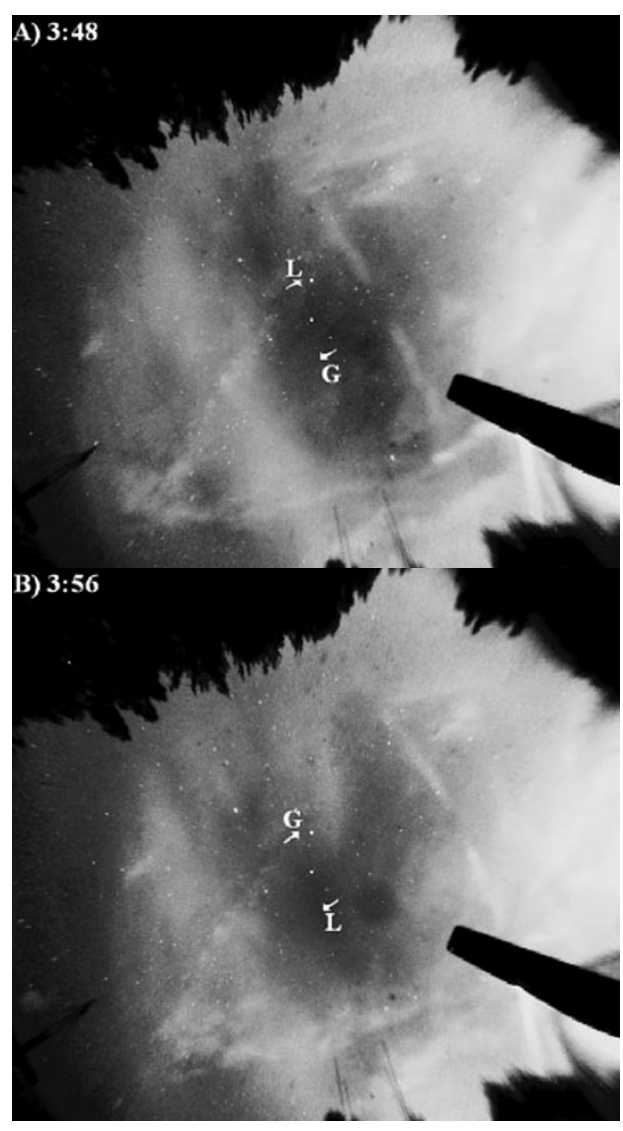

Fig. 11. The allsky frames corresponding to A and B in Fig. 7. L and G show the location of the line feed and Gregorian beams, respectively.

tion band at a lower altitude than the southern component.

Figure 12 shows the smoothened ISR beam profiles corresponding to A and B in Figs. 7 and 11. The threshold for calculating the heights of the squares and circles in Fig. 7 is also shown. It is found from the geometry in Fig. 13 that the equation relating the height ratio to the tilt angle is $\alpha=\arctan \left[2 h_{2} / h_{1}-1.7\right]-15^{\circ}$. According to Fig. 12, the maximum height difference is only $319-286=33 \mathrm{~km}$ $\left(h_{2} / h_{1}=\sim 1.12\right)$, which corresponds to a vertical tilt angle of $\sim 12^{\circ}$. Although both tilts are similar in direction, this value is much smaller than the actual dip angle of the magnetic field line which is $\sim 46^{\circ}$ at $\mathrm{AO}$, and since the actual height difference between the squares/circles is usually smaller, it seems that the vertical tilt of the depletion bands are usually even smaller than $12^{\circ}$. An alternative explanation that does not have to exclude the vertical fieldalignment is related to the shape of the structures. For instance, if these bands are like vertically field-aligned elongated bubbles (that are curved at the southern tip), they could appear as vertically tilted less or even in opposite direction, depending on the relative position of the band with respect to the ISR beam as shown in Fig. 13. The same result is observed when the ISR beams are not located at the center of the MSTID band. When the beams are pointing near the edges of the MSTID band, the apparent tilt in the ISR data is less than that of the geomagnetic field-line. This is also likely due to the curvature of the side (E-W) edges of the MSTID bands. Apparently the bands are more field- aligned at the center and less tilted (more horizontal) at the edges.

Finally, the high altitude bands, which vary with the swing period, are indicated with short lines in Fig. 7. Each high altitude band is mainly due to a neighboring band at a particular instant, and not due to the one just overhead. At high altitudes, the tilted beam extends enough horizontally to detect the nearby band. Each high altitude band appears as intensity-inversed with respect to the over-head band that appears at lower altitudes for that particular time instant in the ISR plot. This is because the neighbor of a dark band is an enhanced band and vice versa. This is also confirmed using allsky images and is replicated in the parallel paper (Seker and Mathews, 2008) using a 3D model. The model geometry also explains why these high altitude bands appear thicker even though one would expect to observe several thinner spikes at higher altitudes since the beam sweeps more horizontal arc per unit time.

Also, certain features seen in the allsky images are explained better based on our finding that the band structures are vertically tilted MSTID structures. First, this interpretation agrees with the inherit assumption of field-alignment in the Perkins Instability theory. Second, the bands are actually wider to the south relative to the north, because they reach higher altitudes to the south and the bubble shape expands probably due to vertical inhomogeneity of the Fregion. If the bands are in fact vertically tilted (the tilt being similar to the orientation of the magnetic field line), that provides clues on the generation mechanism also. In the allsky images, the plasma depletion bands appear from the north, meaning they must be originated at lower altitudes, and consequently at higher latitudes as can be seen from the field line geometry in Fig. 1(b). This suggests that these irregularities are possibly seeded by gravity waves that are generated at lower altitudes. On the contrary, plume shaped depletions appearing from the south in the allsky images during geomagnetic storms could be associated with the poleward surge of high-altitude equatorial spread-F bubbles at the magnetic equator. Studies done with the GUVI instrument on the TIMED satellite clearly show that the equatorial ESF plumes might reach mid-latitudes following the magnetic flux tubes (Kelley et al., 2003c; Kil et al., 2004).

Undoubtedly, in order to fully understand these midlatitude MSTID bands, the physical generation mechanism should be understood. It is not trivial to explain the electrodynamics of these dark bands, mainly due to the fact that the actual horizontal and vertical tilt of the bands $\left(\sim 30^{\circ}\right.$ and $\sim 12^{\circ}$ ) is similar to but somewhat different than that of the magnetic field-line $\left(11^{\circ}\right.$ and $46^{\circ}$ respectively). On the other hand, a difference of $20^{\circ}-35^{\circ}$ is relatively small compared to a possible maximum difference of $180^{\circ}$. Consequently, it could be argued that these MSTID bands are not too far from being field-aligned meaning that B-field plays a major role compared to the E-field and the neutral winds at least one of which must also play a minor role in order to explain the deviation from the field lines. In the literature, the most commonly accepted candidate to explain these structures has been the Perkins Instability (Garcia et al., 2000; Zhou et al., 2006) which inherently assumes small-scale field-aligned irregularities and shows that they grow in time 


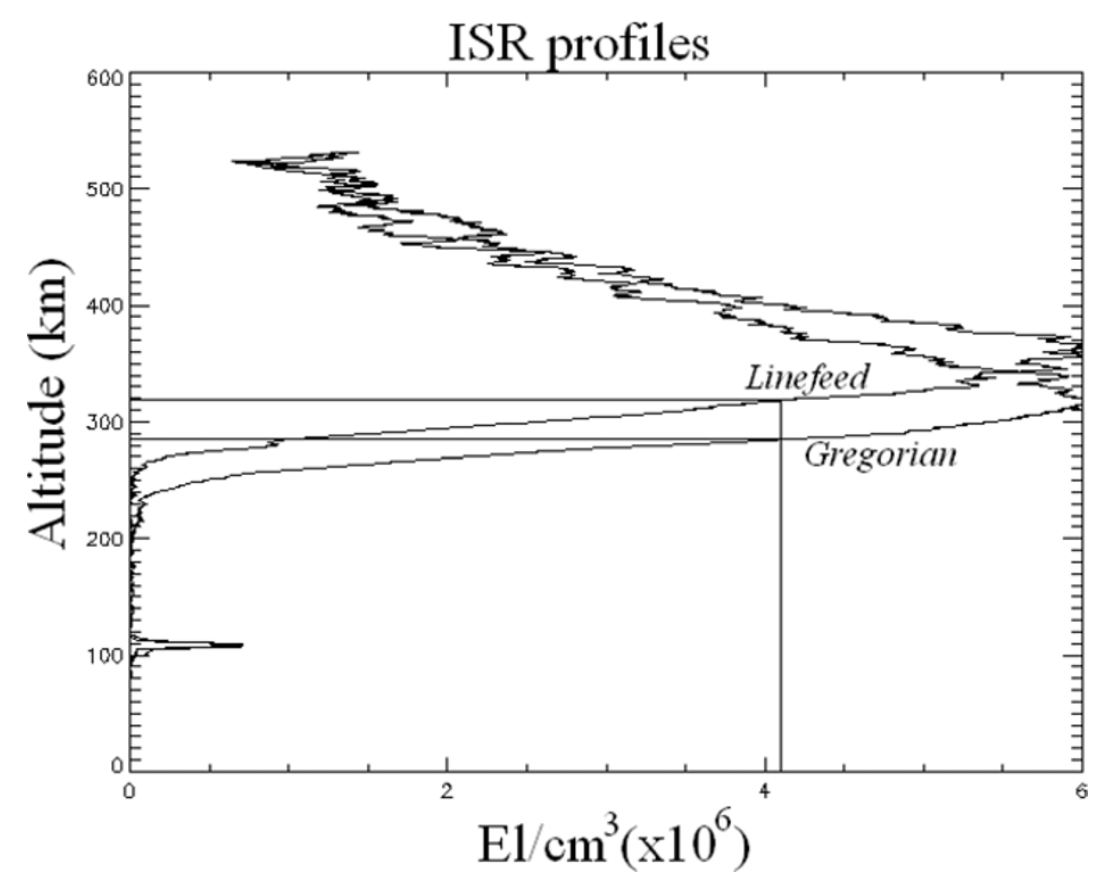

Fig. 12. ISR (linefeed and Gregorian) beam profiles at around 4 am (corresponding to B in Figs. 7 and 11 ). A threshold value of $4.1 \times 10^{6}$ el/cm ${ }^{3}$ ( $\sim 70 \%$ of the maximum) is used to calculate the depletion heights $h_{2}$ and $h_{1}$ that are shown in Fig. 13.

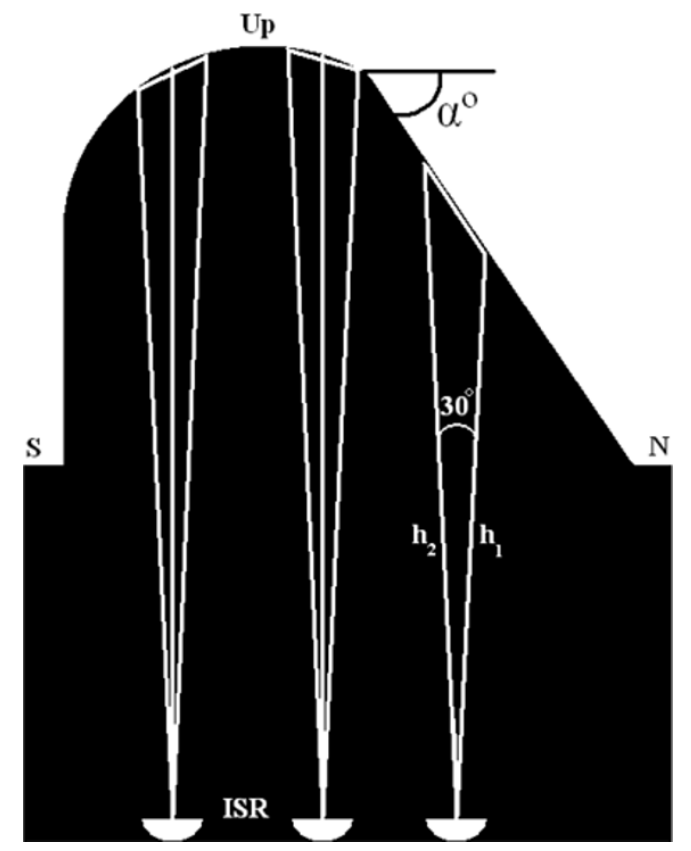

Fig. 13. Sketch of the vertical cross-section of a tilted MSTID band. The geomagnetic north and south are labeled as $\mathrm{N}$ and S, respectively. The three lines represent the zenith and the ISR beam's positions at geomagnetic north and south. Different positions of the ISR relative to the MSTID band result in different apparent tilts due to the curvature of the bubble shape.

in that particular direction for mid-latitudes. It explains the NW-SE alignment of the bands, and-with addition of a polarization electric field (Kelley and Makela, 2001)—SW propagation but its growth rate is too small and independent of scale. As yet unpublished simulations that involve the solution of the Perkins equations in the presence of horizon- tal F-region structure indicates that growth rates are significantly larger in this (expected) situation (Zhou et al., 2008) and match observed growth rates.

However, the Perkins Instability does not involve the necessary seeding mechanism, and thus cannot explain the periodic nature of MSTID bands. Recent papers have linked these structures with gravity waves, based upon their periodic nature. Gravity wave theory can explain the periodicity and the horizontal wavelength; however it cannot explain the very large vertical wavelength of MSTID bands and their directionality, which is explained by the Perkins Instability (Shiokawa et al., 2003a). Based on these results, it appears that a combination of the two theories is the best explanation for the generation of MSTIDs. An alternate seeding mechanism, an oscillating electric field, was also proposed by Shiokawa et al. (2003b). Based on these papers, we believe the MSTID bands presented here are seeded by AGWs at upper E-region altitudes, coupled to plasma at higher altitudes due to ion-neutral collisions, and then this modulated plasma obeys mid-latitude nighttime Fregion electrodynamics (such as the Perkins Instability) at higher altitudes. This explanation emerges as the best so far for our dataset and for mid-latitude MSTID bands in general.

\section{Conclusions}

We have investigated a common phenomenon of midlatitudes-MSTID bands. Combining the observational attributes of an allsky imager, the AO dual-beam ISR and a GPS-TEC system proved to be very useful by revealing that the features seen in the azimuth-scanning ISR data correspond directly to imager and GPS-TEC features and most importantly by additionally providing important clues on the 3D structure of these MSTID bands. These are sum- 
marized below:

1) Packets of several thin "spikes" seen in azimuthscanning ISR are actually created by a single MSTID band.

2) The fork shaped spikes seen in azimuth-scanning ISR data are due to the ISR beam reversing direction and moving in and out of a single MSTID band.

3) The weak, (time wise) thick, high altitude bands (that appear inverse or shifted) with the beam swing period are due to neighboring bands. They appear thicker than expected because the bands expand and merge into the peak of the F-region ionization at higher altitudes.

4) Most importantly, the bands are found to be tilted not only horizontally (aligned northwest-to-southeast), but also vertically as well, with southern parts of a band reaching a higher altitude than the northern part. The tilt is similar to the dip of the magnetic field lines, though the apparent tilt seen in ISR is less than the dip angle. A possible explanation for this would be the curvature at the southern tip and the side edges into an elongated bubble shaped depletion.

Based on these results, we suggest that the geometry of the MSTID bands is "vertically tilted bubbles" with the following parameters: northwest-to-southeast aligned at $\sim 20^{\circ}$ east of the geomagnetic south, depletion "bubbles" tilted $\sim 12^{\circ}$ vertically from horizontal towards geomagnetic south, 50-250 km (geomagnetic) E-W thickness (thicker at higher altitudes, or lower latitudes), 100-200 km horizontal wavelength in the direction of propagation, $100-200 \mathrm{~km} / \mathrm{hr}$ horizontal propagation speed, and $1-\mathrm{hr}$ period. These parameters are consistent with previous studies (e.g. Shiokawa et al., 2003a). Since the bands are vertically tilted and have the bubble shape, the vertical wavelength seems to vary. As shown in Fig. 13, the lower edge of the bands seem to merge with the depleted E-region at $\sim 200 \mathrm{~km}$, otherwise enhanced plasma regions would have been observed below the spikes in the ISR data. Also, the upper edge appears to reach from $250 \mathrm{~km}$ up to $>400 \mathrm{~km}$ depending on the position of the band relative to the ISR beam due to the curvature of the southern tip and the sides of the "bubble" as demonstrated in Fig. 13.

We also discussed what we suspect is the most likely generation mechanism of these mid-latitude MSTID bands: gravity wave seeded Perkins Instability. However, extensive theoretical and modeling studies should be done to further strengthen this claim. In a parallel paper (Seker and Mathews, 2008) we introduce a 3D model of these MSTID bands based on the observations presented here. Using a hypothetical azimuth-scanning ISR, it is further confirmed that these MSTID bands are most likely vertically field-aligned. The synthesized ISR results from this model also confirm all of the conclusions outlined above. We are further improving the model by adding the bubble shape and basing it on theory instead of just observations.

Acknowledgments. This effort was supported under NSF Grants ATM 04-13009 to The Pennsylvania State University. The Arecibo Observatory is part of the National Astronomy and Ionosphere Center which is operated by Cornell University under cooperative agreement with the National Science Foundation. As always, we thank the Arecibo staff for their efforts. Special thanks to Nestor Aponte at Arecibo Observatory for providing the 14-18 June 2004 ISR data.

\section{References}

Aponte, N., S. A. Gonzalez, M. C. Kelley, C. A. Tepley, X. Pi, and B. Iijima, Advection of the equatorial anomaly over Arecibo by smallstorm related disturbance dynamo electric fields, Geophys. Res. Lett., 27, 2833-2836, 2000.

Colerico, M. J., M. Mendillo, D. Nottingham, J. Baumgardner, J. Meriwether, J. Mirick, B. W. Reinisch, J. L. Scali, C. G. Fesen, and M. A. Biondi, Coordinated measurements of $\mathrm{F}$ region dynamic related to the thermospheric midnight temperature maximum, J. Geophys. Res., 101, 26783-26793, 1996.

Garcia, F. J., M. C. Kelley, J. J. Makela, and C.-S. Huang, Airglow observations of mesoscale low-velocity traveling ionospheric disturbances at midlatitudes, J. Geophys. Res., 105, 18407-18415, 2000.

Herrero, F. A. and J. W. Meriwether, 6300- $\AA$ airglow meridional intensity gradients, J. Geophys. Res., 85, 4191-4204, 1980.

Kelley, M. C. and J. J. Makela, Resolution of the discrepancy between experiment and theory of midlatitude F-region structures, Geophys. Res. Lett., 28, 2589-2592, 2001.

Kelley, M. C., J. J. Makela, W. E. Swartz, S. C. Collins, S. Thonnard, N. Aponte, and C. A. Tepley, Caribbean ionosphere campaign, year one: Airglow and plasma observations during two intense mid-latitude spread-F events, Geophys. Res. Lett., 27, 2825-2828, 2000a.

Kelley, M. C., J. J. Makela, and A. Saito, On the electrical structure of airglow depletion/height layer bands over Arecibo, Geophys. Res. Lett., 27, 2837-2840, 2000b.

Kelley, M. C., J. J. Makela, and A. Saito, The mid-latitude F region at the mesoscale: some progress at last, J. Atmos. Solar-Terr. Phys., 64, 15251529, 2002.

Kelley, M. C., C. Haldoupis, M. J. Nicolls, J. J. Makela, A. Belehaki, S. Shalimov, and V. K. Wong, Case studies of coupling between the E and $\mathrm{F}$ regions during unstable sporadic-E conditions, J. Geophys. Res., 108(A12), 1447, doi:10.1029/2003JA009955, 2003a.

Kelley, M. C., J. J. Makela, M. N. Vlasov, and A. Sur, Further studies of the Perkins stability during space weather month, J. Atmos. Solar-Terr. Phys., 65, 1071-1075, 2003b.

Kelley, M. C., J. J. Makela, L. J. Paxton, F. Kamalabadi, J. M. Comberiate, and H. Kil, The first coordinated ground- and space-based optical observations of equatorial plasma bubbles, Geophys. Res. Lett., 30(14), 1766, doi:10.1029/2003GL017301, 2003c.

Kelley, M. C., W. E. Swartz, and J. J. Makela, Mid-latitude ionospheric fluctuation spectra due to secondary ExB instabilities, J. Atmos. and Solar-Terr. Phys., 66(17), 1559-1565, 2004.

Kil, H., S.-Y. Su, L. J. Paxton, B. C. Wolven, Y. Zhang, D. Morrison, and H. C. Yeh, Coincident equatorial ubble detection by TIMED/GUVI and ROSCAT-1, Geophys. Res. Lett., 31, L03809, doi: 10.1029/2003GL018696, 2004.

Link, R. and L. L. Cogger, A reexamination of the OI 6300-Å nightglow, J. Geophys. Res., 93, 9883, 1988.

Livneh, D. J., I. Seker, J. D. Mathews, and F. T. Djuth, Continuous quasiperiodic thermospheric waves over Arecibo, J. Geophys. Res., 112, A07313, doi:10.1029/2006JA012225, 2007.

Makela, J. J., A review of imaging low-latitude ionospheric irregularity processes, J. Atmos. Solar-Terr. Phys., 68, 1441-1458, 2006.

Makela, J. J., S. A. González, B. MacPherson, X. Pi, M. C. Kelley, and P. J. Sultan, Intercomparisons of total electron content measurements using the Arecibo incoherent scatter radar and GPS, Geophys. Res. Lett., 27, 2841-2844, 2000.

Makela, J. J., M. C. Kelley, J. J. Sojka, X. Pi, and A. J. Mannucci, GPS normalization and preliminary modeling results of total electron content during a midlatitude space weather event, Radio Sci., 36, 351-361, $2001 \mathrm{a}$.

Makela, J. J., M. C. Kelley, S. A. González, N. Aponte, and R. P. McCoy, Ionospheric topography maps using multiple-wavelength all-sky images, J. Geophys. Res., 106, 29161-29174, 2001 b.

Mathews, J. D., The incoherent scatter radar as a tool for studying the ionospheric D region, J. Atmos. Terr. Phys., 46, 975-986, 1984.

Mathews, J. D., Incoherent scatter radar probing of the $60-100 \mathrm{~km}$ atmosphere and ionosphere, IEEE Trans. Geosci. Remote Sensing, GE-24, 765-776, 1986.

Mathews, J. D., D. W. Machuga, and Q. Zhou, Evidence for electrodynamic linkages between spread-F, ion rain, the intermediate layer, and 
sporadic E: results from observations and simulations, J. Atmos. SolarTerr. Phys., 63, 1529-1543, 2001.

Mendillo, M., J. Baumgardner, D. Nottingham, J. Aarons, B. Reinisch, J. Scali, and M. C. Kelley, Investigations of thermospheric-ionospheric dynamics with 6300-Å images from the Arecibo Observatory, J. Geophys. Res., 102, 7331-7343, 1997.

Nelson, G. J. and L. L. Cogger, Dynamical behavior of the nighttime ionosphere over Arecibo, J. Atmos. Terr. Phys., 33, 1711, 1971.

Otsuka, Y., T. Kadota, K. Shiokawa, T. Ogawa, S. Kawamura, S. Fukao, and S.-R. Zhang, Optical and radio measurements of a 630-nm airglow enhancement over Japan on 9 September 1999, J. Geophys. Res., 108(A6), 1252, doi:10.1029/2002JA009594, 2003.

Perkins, F. W., Spread F and ionospheric currents, J. Geophys. Res., 78, 218-226, 1973.

Pi, X., M. Mendillo, W. J. Hughes, M. J. Buonsanto, D. P. Sipler, J. Kelly, Q. Zhou, G. Lu, and T. J. Hughes, Dynamical effects of geomagnetic storms and substorms in the middle-latitude ionosphere: An observational campaign, J. Geophys. Res., 105, 7403-7417, 2000.

Sahai, Y., P. R. Fagundes, and J. A. Bittencourt, Transequatorial F-region ionospheric plasma bubbles, J. Atmos. Solar-Terr. Phys., 62, 1377-1383, 2000.

Seker, I. and J. D. Mathews, A 3D model of the MSTID bands based on the incoherent scatter radar and allsky imaging observations, 2008 (in progress).

Seker, I., J. D. Mathews, J. Wiig, P. F. Gutierrez, J. S. Friedman, and C. A. Tepley, First results from the Penn state allsky Imager at Arecibo observatory, Earth Planets Space, 59, 165-176, 2007.

Shiokawa, K., C. Ihara, Y. Otsuka, and T. Ogawa, Statistical study of nighttime medium-scale traveling ionospheric distrurbances using midlatitude airglow images, J. Geophys. Res., 108(A1), 1052, doi:10. 1029/2002JA009491, 2003a.

Shiokawa, K., Y. Otsuka, C. Ihara, T. Ogawa, and F. J. Rich, Ground and satellite observations of nighttime medium-scale traveling iono- spheric disturbance at midlatitude, J. Geophys. Res., 108(A4), 1145, doi: 10.1029/2002JA009639, 2003b.

Swartz, W. E., S. C. Collins, M. C. Kelley, J. J. Makela, E. Kudeki, S Franke, J. Urbina, N. Aponte, S. Gonzalez, M. P. Sulzer, and J. S. Friedman, First observations of an F-region turbulent upwelling coincident with severe E-region plasma and neutral atmosphere perturbations, $J$. Atmos. Solar-Terr. Phys., 64, 1545-1556, 2002.

Taylor, M. J., J.-M. Jahn, S. Fukao, and A. Saito, Possible evidence of gravity wave coupling into the mid-latitude $\mathrm{F}$ region ionosphere during the SEEK campaign, Geophys. Res. Lett., 25, 1801-1804, 1998.

Vadas, S. L., Horizontal and vertical propagation and dissipation of gravity waves in the thermosphere from lower atmospheric and thermospheric sources, J. Geophys. Res., 112, A06305, doi:10.1029/2006JA011845, 2007.

Zhou, Q.-N. and J. D. Mathews, On the physical explanation of the Perkins instability, J. Geophys. Res., 111, A12309, doi:10.1029/2006JA011696, 2006.

Zhou, Q.-N., J. D. Mathews, Q. Du, and C. A. Miller, A preliminary investigation of the pseudo-spectral method numerical solution of the Perkins instability equations in the homogeneous case, J. Atmos. SolarTerr. Phys., 67, 325-335, 2005.

Zhou, Q.-N., J. D. Mathews, C. A. Miller, and I. Seker, The evolution of nighttime mid-latitude mesoscale F-region structures: A case study utilizing numerical solution of the Perkins instability equations, Planet. Space Sci., 54, 710-718, 2006.

Zhou, Q.-N., J. D. Mathews, and Q. Du, Linear stage theoretical and numerical solutions of the Perkins instability equations in the inhomogeneous TEC case, Planet. Space Sci., 2008 (in review).

I. Seker (e-mail: ius102@psu.edu), D. J. Livneh, J. J. Makela, and J. D. Mathews 